\title{
Blind identification strategies for room occupancy estimation
}

\author{
A. Ebadat, G. Bottegal, D. Varagnolo, \\ B. Wahlberg, Fellow, IEEE, H. Hjalmarsson, Fellow, IEEE, and K. H. Johansson, Fellow, IEEE
}

\begin{abstract}
We propose and test on real data a two-tier estimation strategy for inferring occupancy levels from measurements of $\mathrm{CO}_{2}$ concentration and temperature levels. The first tier is a blind identification step, based either on a frequentist Maximum Likelihood method, implemented using non-linear optimization, or on a Bayesian marginal likelihood method, implemented using a dedicated Expectation-Maximization algorithm. The second tier resolves the ambiguity of the unknown multiplicative factor, and returns the final estimate of the occupancy levels.

The overall procedure addresses some practical issues of existing occupancy estimation strategies. More specifically, first it does not require the installation of special hardware, since it uses measurements that are typically available in many buildings. Second, it does not require apriori knowledge on the physical parameters of the building, since it performs system identification steps. Third, it does not require pilot data containing measured real occupancy patterns (i.e., physically counting people for some periods, a typically expensive and time consuming step), since the identification steps are blind.
\end{abstract}

Index Terms - System identification, management of HVAC systems, Maximum Likelihood, Expectation-Maximization

\section{INTRODUCTION}

Estimating occupancy levels in the built environment is an important task both for the control of Heating, Venting and Air Conditioning (HVAC) systems and for diagnostic purposes [1], [2], [3], [4]. There are several approaches to address occupancy estimation: $i$ ) use dedicated hardware for counting people, [5], [6], [7]; ii) use only information from non-dedicated hardware, already installed in the building or iii) combine information from both new and pre-existing hardware. Unfortunately, most of the buildings are not equipped with dedicated occupancy counting hardware, and installing new hardware is expensive and time consuming. There is thus a general interest in understanding how we can obtain occupancy estimates exploiting basic and generally available information, such as the $\mathrm{CO}_{2}$ concentration and temperaturs of the various rooms.

A. Ebadat, G. Bottegal, B. Wahlberg, H. Hjalmarsson and K. H. Johansson are with ACCESS the Department of Automatic Control, School of Electrical engineering, KTH Royal Institute of Technology, Sweden. Emails: \{ ebadat | bottegal | bo | hjalmars I kallej \}@kth.se. D. Varagnolo is with the Division of Signals and Systems, Department of Computer Science, Electrical and Space Engineering, Luleå University of Innovation and Technology, Luleå, Sweden. Email: damiano.varagnoloeltu.se.

This work is supported by the European Institute of Technology (EIT) Information and Communication Technology (ICT) Labs, the Swedish Energy Agency, the Swedish Governmental Agency for Innovation Systems (VINNOVA), the Knut and Alice Wallenberg Foundation, the Framework Programme for Research and Innovation Horizon 2020 under grant agreement n. 636834 (DISIRE), and the Swedish research council Norrbottens Forskningsråd
Literature review: Inferring occupancy levels from environmental signals such as $\mathrm{CO}_{2}$ levels and temperatures requires the deployment of models that describe the effects of occupancy on $\mathrm{CO}_{2}$ and temperatures. These models can then be obtained by means of either considerations involving first principles and physical laws, or from data-driven techniques. We hereby focus on the latter choice. Data driven techniques typically make use black-box appraoches and do not require additional knowledge on the physics underlying the phenomenon. The model is carried out using opportune learning techniques (Support Vector Machines (SVMs), Neural Networks (NNs), Hidden Markov Models (HMMs), system identification methods, etc.). This usually requires collection of training datasets containing both environmental signals and occupancy levels, meaning that the true occupancy has to be measured for a certain time. Under this umbrella we can group methods based on electricity consumptions levels [8], $\mathrm{CO}_{2}$-based features (e.g., averages of the signals in time, first or second-order temporal differences) [9], [10], [11], measurements from Passive Infrared (PIR) sensors [12], [13], activity of the Information Technology (IT) infrastructure such as access to the Internet [14].

The main drawback of the aforementioned data-driven techniques is the need of a training set comprising occupancy measurements. Collecting these measurements might be expensive or not feasible, even for short times. Then, the natural question is whether we can train models blindly, i.e., identify models using training sets that do not include measurements of the occupancy. An answer to this question can be provided by blind identification methods [15].

Statement of contributions: The object of this paper is to study how we can estimate occupancy levels in a room using information of $\mathrm{CO}_{2}$ concentration levels and ventilation only. We shall not consider the temperature signal, as it does not provide significant insights into the occupancy levels. We propose a novel estimator consisting of two tiers. Tier- 1 is a blind identification step based on either a frequentist Maximum Likelihood (ML) method or on the empirical Bayes method. The outcome of tier-1 is, up to a multiplicative factor, both occupancy levels and a model for the $\mathrm{CO}_{2}$ dynamics. Tier-2 consists of strategy to compute the unknown multiplicative factor that best explains the data and finalizes the occupancy estimation problem. The clear advantage of our technique is that we do not require any knowledge of the physical parameters of the built environment (required when constructing model-based estimators) and we do not need to measure true occupancy levels for a 
certain period of time (required when constructing non-blind data-driven estimators).

Organization of the manuscript: Section II describes the underlying assumptions and formulates the problem in mathematical terms. Section III presents the proposed blind identification technique. Section IV elaborates on the second tier of the algorithm. Section V evaluates the effectiveness of the strategy on some real-world dataset, and Section VI summarizes our remarks and ideas for future developments.

\section{ASSUMPTIONS AND PROBLEM FORMULATION}

In this paper we make the following assumptions:

A1) from a properly equipped room we measure:

- $y(t) \in \mathbb{R}$, the $\mathrm{CO}_{2}$ concentration levels in the room;

- $u(t) \in \mathbb{R}$, the actuation levels of the HVAC system, i.e., how much fresh air is injected in the room;

- (optional, not strictly required for constructing the estimation strategy) $d(t) \in\{0,1\}$, whether the door of the room has been opened or not;

A2) the time domain of the signals is discrete and finite; w.l.o.g., the sampling time is 1 and the window length is $N$;

A3) as in [16], [17], [18], [19], [20], the structural form of the dynamics of the $\mathrm{CO}_{2}$ concentration levels $y(t)$ are described by the generic Linear Time Invariant (LTI) model

$$
y(t)=\left[g_{y} * y\right](t)+\left[g_{u} * u\right](t)+\left[g_{o} * o\right](t)+e(t)
$$

where $*$ denotes convolution, $g_{y}, g_{u}$ and $g_{o}$ are strictly causal BIBO-stable impulse responses, $o(t)$ represents the occupancy pattern, i.e., how many occupants are in the room at each time instant, and $e(t)$ is a Gaussian white noise with unknown variance $\sigma^{2}$;

A4) the unique available priors for the occupancy levels are:

A4i) $o(t) \in \mathbb{N}_{+}$and $o(t)-o(t-1)$ is sparse, i.e., the occupancy levels are positive natural numbers and tend to remain constant for given periods of time;

A4ii) the maximal variation of the occupancy levels is bounded and known, i.e., $|o(t)-o(t-1)| \leq \gamma_{\max }$ for all $t=1, \ldots, N$ and for a known $\gamma_{\max }$ (this knowledge can be obtained, e.g., by practical considerations on the size and usage of the room);

A5) (optional, not strictly required for constructing the estimation strategy) thanks to the knowledge of the door opening signal $d(t)$, we know the time instants $t_{1}, \ldots, t_{M}$ at which the door is open (and thus at which the occupancy signal $o(t)$ potentially changes). The knowledge of $d(t)$ is summarized by the knowledge of the constraints

$$
\begin{gathered}
o(1)=\ldots=o\left(t_{1}-1\right), \\
o\left(t_{1}\right)=\ldots=o\left(t_{2}-1\right), \\
\vdots \\
o\left(t_{M}\right)=\ldots=o(N) .
\end{gathered}
$$

Letting $t_{0}:=1$, this implies that estimating $\left[o\left(t_{0}\right), o\left(t_{1}\right), \ldots, o\left(t_{M}\right)\right]$ is equivalent to estimate $[o(1), o(2), \ldots, o(N)]$.

The considered problem is then to develop a strategy that estimates the occupancy levels $\left[o(1), o\left(t_{1}\right), \ldots, o\left(t_{M}\right)\right]$ using the available information. In other words, given the facts that $y(1), y(2), \ldots$ and $u(1), u(2), \ldots$ are measured and known, and that $g_{y}, g_{u}$ and $g_{o}$ are unknown, estimate $o(1), o(2), \ldots$.

\section{BLIND SYSTEM IDENTIFICATION ALGORITHMS}

Considering dynamics (1), it is immediate to recognize that both signals $g_{o}(t)$ and $o(t)$ are unknown. Since they appear as a convolution, the problem of estimating the couple $g_{o}$ and $o$ is ill-posed, in the sense that:

Remark 1 (non-identifiability issue) Let $\widetilde{g}_{o}=\frac{1}{\alpha} g_{o}$ and $\widetilde{o}=$ $\alpha o$. Then $\widetilde{g}_{o} \widetilde{o}=g_{o} o$, for every $\alpha$.

This non-identifiability problem is typical of blind identification frameworks. We thus devise the existence of two tiers: the first is the blind problem of estimating $g_{y}, g_{u}, g_{o}$ and $o$ (the last two up to the multiplicative factor above) from the available information. The second is of estimating the "best" $\alpha$ from the same data used for estimating $o$.

Postponing the second tier to Section IV, we focus now on the blind identification step. We then propose 2 different solutions for this identification process: a frequentist ML approach in Section III-B, and a Bayesian marginal likelihood approach implemented using Expectation-Maximization (EM) algorithm in Section IIIC. Before proceeding we collect some notation for ease of reference.

\section{A. Additional notation}

Define

$$
\begin{gathered}
\boldsymbol{y}:=\left[\begin{array}{c}
y(1) \\
\vdots \\
y(N)
\end{array}\right] \boldsymbol{u}:=\left[\begin{array}{c}
u(1) \\
\vdots \\
u(N)
\end{array}\right] \boldsymbol{o}:=\left[\begin{array}{c}
o(1) \\
\vdots \\
o(N)
\end{array}\right] \boldsymbol{e}:=\left[\begin{array}{c}
e(1) \\
\vdots \\
e(N)
\end{array}\right] \\
\boldsymbol{U}:=\left[\begin{array}{ccccc}
u(1) & 0 & 0 & \ldots & 0 \\
u(2) & u(1) & \ldots & \ddots & 0 \\
\vdots & \vdots & &
\end{array}\right]
\end{gathered}
$$

Similarly to $\boldsymbol{U}$, we also define $\boldsymbol{O}$ and $\boldsymbol{Y}$ from the signals $o(t)$ and $y(t)$, respectively. Moreover, from the impulse responses defining dynamics (1) we define

$$
\boldsymbol{g}_{y}:=\left[\begin{array}{c}
0 \\
g_{y}(1) \\
\vdots \\
g_{y}(n)
\end{array}\right] \quad \boldsymbol{g}_{u}:=\left[\begin{array}{c}
0 \\
g_{u}(1) \\
\vdots \\
g_{u}(n)
\end{array}\right] \quad \boldsymbol{g}_{o}:=\left[\begin{array}{c}
0 \\
g_{o}(1) \\
\vdots \\
g_{o}(n)
\end{array}\right]
$$

which are the vector of truncated impulse response coefficients. The integer $n$ is such that the dynamics defined by $\boldsymbol{g}_{y}, \boldsymbol{g}_{u}, \boldsymbol{g}_{o}$ approximate well the dynamics defined by 
$g_{y}(1), g_{y}(2), \ldots, g_{u}(1), g_{u}(2), \ldots$ and $g_{o}(1), g_{o}(2), \ldots$ We also use

$$
\Delta:=\left[\begin{array}{ccc}
0 & \cdots & 0 \\
I_{N-1} & \vdots \\
& & 0
\end{array}\right]
$$

(with $I_{N-1}$ the $N-1$-dimensional identity matrix) to perform time-shift operations on the previous vectors.

To summarize: $\boldsymbol{y}$ and $\boldsymbol{u}$ are measured and unknown; $\boldsymbol{o}$, $\boldsymbol{g}_{y}, \boldsymbol{g}_{u}$ and $\boldsymbol{g}_{o}$ are unknown; $\boldsymbol{o}$ is to be estimated.

\section{B. A frequentist ML approach}

As suggested in [21], [20], let the dynamics (1) correspond to the first-order AutoRegressive eXogenus (ARX) system

$$
y(t)=a y(t-1)+b_{u} u(t-1)+b_{o} o(t-1)+e(t),
$$

where $e(t)$ is white Gaussian with unknown variance $\sigma^{2}$, or, in compact form, let

$\boldsymbol{y}=(I-a \Delta)^{-1} b_{u} \Delta \boldsymbol{u}+(I-a \Delta)^{-1} b_{o} \Delta \boldsymbol{o}+(I-a \Delta)^{-1} \boldsymbol{e}$.

Let then the (in this subsection deterministic) vector of the unknown model parameters and occupancy levels be collected in

$$
\theta_{\mathrm{ARX}}:=\left[\begin{array}{lllllll}
a & b_{u} & b_{o} & \sigma^{2} & o\left(t_{0}\right) & \ldots & o\left(t_{M}\right)
\end{array}\right]^{T}
$$

Since $e(t) \sim \mathcal{N}\left(0, \sigma^{2}\right)$, it follows that the likelihood function is

$$
p\left(\boldsymbol{y} ; \theta_{\mathrm{ARX}}\right) \sim \mathcal{N}\left(m_{y}, \Sigma_{y}\right)
$$

with

$$
\left\{\begin{array}{l}
m_{y}=(I-a \Delta)^{-1} b_{u} \Delta \boldsymbol{u}+(I-a \Delta)^{-1} b_{o} \Delta \boldsymbol{o} \\
\Sigma_{y}=\sigma^{2}(I-a \Delta)^{-1}(I-a \Delta)^{-T}
\end{array}\right.
$$

Given the priors assumed in Assumption A4, we can use an $\mathrm{ML}$ approach to estimate the unknown $\theta_{\mathrm{ARX}}$, i.e., estimate the unknown as $\widehat{\theta}_{\mathrm{ARX}}:=\arg \max _{\theta} p(\boldsymbol{y} ; \theta)$. Computing the $\mathrm{ML}$ estimate is then equivalent to solve the optimization problem

$$
\begin{array}{ll}
\min _{\theta_{\mathrm{ARX}}} & L\left(\theta_{\mathrm{ARX}}\right) \\
\text { s.t. } & o\left(t_{i}\right) \in \mathbb{N}_{+} \quad i=0, \ldots, M \\
& 0<a<1
\end{array}
$$

with $L\left(\theta_{\text {ARX }}\right)$ the log-likelihood, i.e.,

$$
\begin{gathered}
L\left(\theta_{\mathrm{ARX}}\right):=\log \operatorname{det}\left(\Sigma_{y}\right)+\frac{\overline{\boldsymbol{y}}^{T} \overline{\boldsymbol{y}}}{\sigma^{2}}, \\
\overline{\boldsymbol{y}}:=(I-a \Delta) \boldsymbol{y}-b_{u} \Delta \boldsymbol{u}-b_{o} \Delta \boldsymbol{o} .
\end{gathered}
$$

Notice that the constraint $0<a<1$ in (10) ensures stable dynamics. Since problem (10) is mixed-integer nonlinear, its solution requires the implementation of appropriate numerical schemes. To ease the computational requirements, we relax the constraint $o\left(t_{i}\right) \in \mathbb{N}_{+}$into $o\left(t_{i}\right) \in \mathbb{R}_{+}$, and propose to solve the relaxed problem using commercial non-linear constrained optimization tools (e.g., fmincon in Matlab). Hence, our blind estimation problem becomes

$$
\begin{array}{ll}
\min _{\theta_{\mathrm{ARX}}} & L\left(\theta_{\mathrm{ARX}}\right) \\
\text { s.t. } & 0<a<1 .
\end{array}
$$

Due to the presence of a term $1 / \sigma^{2}$, problem (11) is highly non-linear and still numerically demanding. To further diminish the computational burden it is then possible to exploit an alternated approach which iterates between optimizing the model parameters and the occupancy vector, i.e., alternate the two steps, each of reduced computational complexity: 1) fix the model parameters, and estimate the occupancy signal given that parameters. The reduced optimization problem in this case is quadratic, and can be easily solved by standard optimization routines; 2) fix the occupancy signal, and estimate the model parameters. Iterating these two steps converges then to the ML solution [22], [23].

\section{A Bayesian marginal likelihood approach}

Exploiting the notation of Section III-A, we can rewrite an approximation of (1), in vector form as

$$
\boldsymbol{y}=\boldsymbol{Y} \boldsymbol{g}_{y}+\boldsymbol{U} \boldsymbol{g}_{u}+\boldsymbol{O} \boldsymbol{g}_{o}+\boldsymbol{e} .
$$

Consider then Bayesian models for the impulse responses $\boldsymbol{g}_{y}, \boldsymbol{g}_{u}$ and $\boldsymbol{g}_{o}$. More specifically, conforming to the current trend in the system identification community [24], [25], [26], consider Gaussian priors of the type

$$
\begin{aligned}
p\left(\boldsymbol{g}_{y} ; \lambda_{y}, \beta_{y}\right) & =\mathcal{N}\left(0, \lambda_{y} K_{\beta_{y}}\right) \\
p\left(\boldsymbol{g}_{u} ; \lambda_{u}, \beta_{u}\right) & =\mathcal{N}\left(0, \lambda_{u} K_{\beta_{u}}\right) \\
p\left(\boldsymbol{g}_{o} ; \beta_{o}\right) & =\mathcal{N}\left(0, K_{\beta_{o}}\right) .
\end{aligned}
$$

with $K_{\beta_{*}}$ representing a covariance matrix (or kernel) drawn from the family of stable spline kernels [25], [24], [26], In this paper, we make use of the first-order stable spline kernels, defined as

$$
\left[K_{\beta}\right]_{i, j}:=\beta^{\max (i, j)} \quad 0<\beta<1 .
$$

This kernel depends on the hyperparameter $\beta$, which regulates how fast realizations drawn from such a prior decay in time. Note that $p\left(\boldsymbol{g}_{y} ; \lambda_{y}, \beta_{y}\right)$ and $p\left(\boldsymbol{g}_{u} ; \lambda_{u}, \beta_{u}\right)$ depend also on the scaling factors $\lambda_{y}$ and $\lambda_{u}$, which tune the amplitude of the unknown impulse responses. This parameter is not required in the prior of $\boldsymbol{g}_{o}$, since in this case, due to the intrinsic non-identifiability of blind identification problems, it is meaningless to determine it.

Defining then the unknown parameters as

$$
\theta:=\left[\begin{array}{lllllllll}
o\left(t_{0}\right) & \ldots & o\left(t_{M}\right) & \beta_{y} & \lambda_{y} & \beta_{u} & \lambda_{u} & \beta_{o} & \sigma^{2}
\end{array}\right]
$$

a natural approach to estimate $\theta$ is then to maximize the marginal likelihood of the output with respect to $\theta$, namely

$$
\widehat{\theta}:=\arg \max _{\theta} \log p(\boldsymbol{y} ; \theta),
$$

which is obtained by integrating out the dependence on the unknown impulse responses $\boldsymbol{g}_{y}, \boldsymbol{g}_{u}$ and $\boldsymbol{g}_{o}$ from the joint probability $p\left(\boldsymbol{y}, \boldsymbol{g}_{y}, \boldsymbol{g}_{u}, \boldsymbol{g}_{o} ; \theta\right)$.

Being the numerical solution of (16) not trivial, we then resort to an EM scheme. Since

$$
p(\boldsymbol{y} ; \theta)=\frac{p\left(\boldsymbol{y}, \boldsymbol{g}_{y}, \boldsymbol{g}_{u}, \boldsymbol{g}_{o} ; \theta\right)}{p\left(\boldsymbol{g}_{y}, \boldsymbol{g}_{u}, \boldsymbol{g}_{o} \mid \boldsymbol{y} ; \theta\right)},
$$


the estimate $\widehat{\theta}_{\mathrm{EM}}$ of $\theta_{\mathrm{EM}}$ can be computed by iterating the two following steps (with $k$ the iteration index):

E step: given

$$
\widehat{\theta}_{k}:=\left[\begin{array}{lllllll}
o_{k}\left(t_{0}\right) & \ldots & o_{k}\left(t_{M}\right) & \beta_{o, k} & \lambda_{k} & \beta_{u, k} & \sigma_{k}^{2}
\end{array}\right],
$$

i.e., the estimate of $\widehat{\theta}_{\mathrm{EM}}$ at iteration $k$, compute

$$
Q\left(\theta, \widehat{\theta}_{k}\right)=\mathbb{E}_{p\left(\boldsymbol{g}_{y}, \boldsymbol{g}_{u}, \boldsymbol{g}_{o} \mid \boldsymbol{y} ; \widehat{\theta}_{k}\right)}\left[\log p\left(\boldsymbol{y}, \boldsymbol{g}_{y}, \boldsymbol{g}_{u}, \boldsymbol{g}_{o} ; \theta\right)\right] ;
$$

\section{M step: compute}

$$
\widehat{\theta}_{k+1}=\arg \max _{\theta} Q\left(\theta, \widehat{\theta}_{k}\right) .
$$

The general convergence properties of the EM algorithm assure then that by iterating the two previous steps $\widehat{\theta}_{k}$ asymptotically converges to a (potentially local) maximum of the marginal likelihood $p(\boldsymbol{y} ; \theta)$. The procedure is stopped either when $\left\|\widehat{\theta}_{k+1}-\widehat{\theta}_{k}\right\|$ is below a given threshold or after a pre-fixed number of iterations. We refer to [27] for details on the implementation of the EM scheme.

\section{SOLVING THE NON-IDENTIFIABILITY ISSUE}

As stated by Remark 1, solving either (11) or (16) does not solve completely the problem of estimating occupancy levels, since the estimates $\widehat{g}_{o}$ and $\widehat{o}$ are only determined up to a scaling factor. In other words, after solving (11) or (16), one obtains the family of estimates $\frac{1}{\alpha} \widehat{g}_{o}$ and $\alpha \widehat{o}$ parameterized by $\alpha>0$.

The problem then becomes to find a value $\alpha^{*}$ such that $\alpha^{*} \widehat{o}$ is the best estimator for the true occupancy $o$ in sense defined later.

To compute $\alpha^{*}$ we thus attempt to exploit Assumption A4ii, that is the knowledge (e.g., due to physical constraints or considerations on the usage of the building) that the temporal variations of the true occupancy levels are bounded. Let then

$$
\delta(t):=o(t)-o(t-1), \quad \widehat{\delta}(t):=\widehat{o}(t)-\widehat{o}(t-1),
$$

and consider, by assumption A4ii, we know $\gamma_{\max }$ so that

$$
o(t)-o(t-1)=: \delta(t) \in \Gamma:=\left\{-\gamma_{\max }, \ldots,+\gamma_{\max }\right\} \subset \mathbb{N} .
$$

To better explain our approach to the estimation of $\alpha^{*}$, let us consider the following situations:

1) if the estimates $\widehat{o}$ were perfect, and if we also knew $\alpha^{*}$, then $\alpha^{*} \widehat{\boldsymbol{o}}=\boldsymbol{o}$ would hold. Consequently, $\alpha^{*} \widehat{\delta}(t)=$ $\delta(t)$ would hold, and thus, in this hypothetical case, one would have $\alpha^{*} \widehat{\delta}(t) \in \Gamma$ for each $t$

2) if the estimates $\widehat{o}$ were perfect, but instead we did not know $\alpha^{*}$, then again $\alpha^{*} \widehat{\boldsymbol{o}}=\boldsymbol{o}$ would hold, but in this case one would have to compute $\alpha^{*}$. To compute $\alpha^{*}$ then one may exploit the fact that $\alpha^{*} \widehat{\delta}(t)$ should be in $\Gamma$ for each $t$, as said above.

Denoting then the generic element of $\Gamma$ by $\gamma_{j}$, we know that for each $t$ there should be a corresponding $\gamma_{j}$ so that $\alpha^{*} \widehat{\delta}(t)=\gamma_{j}$, or, equivalently, there exists $\gamma(t) \in \Gamma$ that minimizes $\left(\alpha^{*} \widehat{\delta}(t)-\gamma(t)\right)^{2}$. Considering all the time instances $t$ 's, then, it follows that $\alpha^{*}$ minimizes the element-wise distance between the set $\{\alpha \widehat{\delta}(t)\}$ and $\gamma$, i.e.,

$$
\alpha^{*}=\arg \min _{\substack{\alpha>0 \\ \gamma(t) \in \Gamma}} \sum_{t=2}^{N}(\alpha \widehat{\delta}(t)-\gamma(t))^{2} ;
$$

3 ) in the real-world case, however, we do not know $\alpha^{*}$ and $\widehat{\boldsymbol{o}}$ is imperfect, so that there is no $\alpha$ such that $\alpha^{*} \widehat{\boldsymbol{o}}$ is equal to $\boldsymbol{o}$. Nonetheless it is meaningful to still identify $\alpha^{*}$ as that particular $\alpha$ that minimizes the distance between the set $\{\alpha \widehat{\delta}(t)\}$ and the set $\Gamma$, i.e.,

$$
\alpha^{*}=\arg \min _{\substack{\alpha>0 \\ \gamma(t) \in \Gamma}} \sum_{t=2}^{N}(\alpha \widehat{\delta}(t)-\gamma(t))^{2} .
$$

This implicitly corresponds to assume that each $\alpha^{*} \widehat{\delta}(t)$ is close enough to $\Gamma$ even if the estimates are not perfect.

Unfortunately (21) is a Mixed-Integer Quadratic program that requires, for every $\alpha$, to compute the closest element $\gamma(t) \in \Gamma$ for each $\alpha \widehat{\delta}(t)$.

To relax this association burden problem we then propose to exploit the following ad-hoc classification scheme: for each $\alpha$ and $\widehat{\delta}(t)$ let the associated $\gamma_{\alpha}(t)$ be

$$
\gamma_{\alpha}(t)=\left\{\begin{array}{cl}
-\gamma_{\max } & \text { if } \alpha \widehat{\delta}(t) \leq-\gamma_{\max } \\
{[\alpha \widehat{\delta}(t)]} & \text { if }[\alpha \widehat{\delta}(t)\rfloor \in \Gamma \\
\gamma_{\max } & \text { if } \alpha \widehat{\delta}(t) \geq \gamma_{\max }
\end{array}\right.
$$

(cf. Figure 1 and its caption). After running classifier (22) we can thus relax problem (21) into

$$
\alpha^{*}=\arg \min _{\alpha>0} \sum_{t=2}^{N}\left(\alpha \widehat{\delta}(t)-\gamma_{\alpha}(t)\right)^{2} .
$$

Notice that (23) is not a Least Squares (LS) problem since the decision variable enters also in the classifier $\gamma_{\alpha}(t)$, and is also not convex; however, with $\alpha$ being a scalar (23) can be solved by search over and appropriate grid of $\alpha$-values.

Notice that there is also no guarantee of uniqueness of the solution, and that at the current state of the art there is no statistical analysis of the performance of the strategy (not even if the estimator is consistent). We nonetheless noticed that it seems performing well from practical perspectives, and thus plan to perform analyses as future works.

Remark 2 Having knowledge of true and non-null occupancy levels o $\left(t_{1}^{\prime}\right), \ldots, o\left(t_{k}^{\prime}\right)$ during times $t_{1}^{\prime}, \ldots, t_{k}^{\prime}$ leads to an immediate solution of the non-identifiability issue, that can be used to skip problem (23). Indeed, letting

$$
\widehat{\mathcal{O}}:=\left[\widehat{o}\left(t_{1}^{\prime}\right), \ldots, \widehat{o}\left(t_{k}^{\prime}\right)\right]^{T} \quad \mathcal{O}:=\left[o\left(t_{1}^{\prime}\right), \ldots, o\left(t_{k}^{\prime}\right)\right]^{T},
$$

it is then statistically meaningful to compute $\alpha^{*}$ as the minimizer of the empirical Mean Square Error (MSE) between 


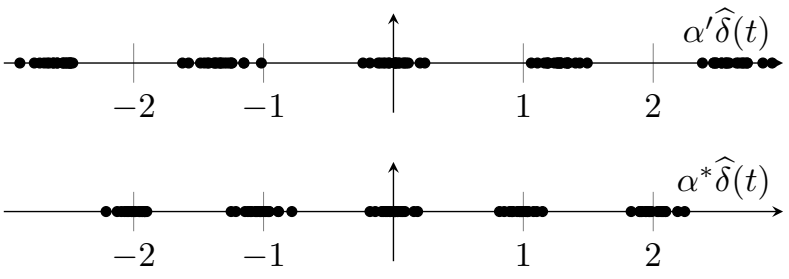

Fig. 1. Exemplification of the proposed mechanism of solving the nonidentifiability issue through classifier (22), with $\Gamma=\{-2, \ldots, 2\}$. The black dots in each panel above represent the various $\widehat{\delta}(t) \mathrm{s}$; they are scaled differently in the two panels because they are pre-multiplied by different $\alpha$ s. The ticks in the $\mathrm{x}$-axes represent instead $\Gamma$. The overall scheme for the computation of $\alpha^{*}$ then works as follows: for each $\alpha$, apply (22) and thus associate to each black dot, i.e., each $\widehat{\delta}(t)$, the closest tick in the $\mathrm{x}$-axis. Compute then for that $\alpha$ the sum of the distances between the various $\widehat{\delta}(t) \mathrm{s}$ and the $\mathrm{x}$-axis ticks. Get then $\alpha^{*}$ as that $\alpha$ that minimizes this sum of distances. In this figure, the top panel presents a suboptimal $\alpha$, while the bottom panel presents the optimal $\alpha^{*}$. Here $\alpha^{\prime}$ is worse than $\alpha^{*}$ since the average element-wise distance of the various $\alpha \widehat{\delta}(t)$ s from $\Gamma$ is bigger than the average one of the various $\alpha^{*} \widehat{\delta}(t) \mathrm{s}$.

the estimates $\alpha \widehat{\mathcal{O}}$ and the real occupancy levels $\mathcal{O}$, i.e., as the solution of the LS problem

$$
\alpha^{*}=\arg \min _{\alpha>0}\|\alpha \widehat{\mathcal{O}}-\mathcal{O}\|^{2}=\left(\widehat{\mathcal{O}}^{T} \widehat{\mathcal{O}}\right)^{-1} \widehat{\mathcal{O}}^{T} \mathcal{O} \text {. }
$$

Notice that the previous problem is well posed only if there exists at least one index in $t_{1}^{\prime}, \ldots, t_{k}^{\prime}$ for which $\widehat{o}(t) \neq 0$ and $o(t) \neq 0$ simultaneously. In other words, one could skip the second tier of the estimation strategy proposed in this paper by collecting information on true occupancy levels just during one instant. This is essentially different from other data-driven techniques, where the process of collecting information on true occupancy levels must be sufficiently long so to capture properly the dynamics of the system.

\section{NUMERICAL EXPERIMENTS}

We test our strategy on the dataset used in [10], enabling the possibility of comparing different strategies on the same benchmark. The dataset, available at http://hvac . ee.kth.se/datasets.html, comprises two weeks of measurements of $\mathrm{CO}_{2}$ and temperature levels from $\mathrm{HDH}$ sensors and measurements of venting, cooling and heating actuation levels from the central HVAC management system, occupancy levels manually registered for the whole period with a time accuracy of 1 minute. Before applying the algorithms proposed in this manuscript signals have been resampled using linear interpolation schemes to intervals of 5 minutes.

To quantitatively measure the effectiveness of the estimation strategies we then consider the following performance indicators: $i$ ) the $M S E$

$$
\operatorname{MSE}(\widehat{\boldsymbol{o}}):=\frac{\|\widehat{\boldsymbol{o}}-\boldsymbol{o}\|_{2}^{2}}{\|\boldsymbol{o}\|_{2}^{2}},
$$

characterizing the relative estimation errors; ii) the accuracy

$$
\operatorname{Acc}(\widehat{\boldsymbol{o}}):=1-\frac{\|\mathbb{1}(\widehat{\boldsymbol{o}}-\boldsymbol{o})\|_{1}}{N},
$$

reporting how many times the estimates are perfect by means of the L1 norm of the indicator function

$$
\mathbb{1}(\boldsymbol{x}):=\left\{\begin{array}{c}
\mathbb{1}(x(1)) \\
\vdots \\
\mathbb{1}(x(N))
\end{array} \quad \mathbb{1}(x(t)):= \begin{cases}1 & \text { if } x(t)>0 \\
0 & \text { otherwise }\end{cases}\right.
$$

iii) the false positive / false negative occupancy detection rates

$$
\mathrm{FP}(\widehat{\boldsymbol{o}}):=\widehat{\beta}(0), \quad \mathrm{FN}(\widehat{\boldsymbol{o}}):=1-\widehat{\beta}(1),
$$

describing the ability of discriminating the presence / absence of occupants in terms of false positives (when the room is estimated to be occupied while it is not) and false negatives (when the room is estimated to be empty while it is not) by means of the empirical power function

$$
\widehat{\beta}(\theta):=\frac{1}{\left|\mathcal{N}_{\theta}\right|} \sum_{k \in \mathcal{N}_{\theta}} \mathbb{1}(\widehat{o}(k)),
$$

in its turn based on the definition of the sets

$$
\mathcal{N}_{\theta}:=\{t \text { s.t. } \mathbb{1}(o(k))=\theta\}, \quad \theta=\{0,1\},
$$

dividing the time indexes in the sets $\mathcal{N}_{0}$, for the $k$ 's for which the room was not occupied, and $\mathcal{N}_{1}$, for the $k$ 's for which the room was occupied.

We then report in Figure 2 the qualitative results of the estimation procedure. Noticeably, the whole procedure is able to detect correctly when the room is not occupied, and detects fairly well also when the room is occupied (even if it is not extremely accurate in understanding how many people is in the room).
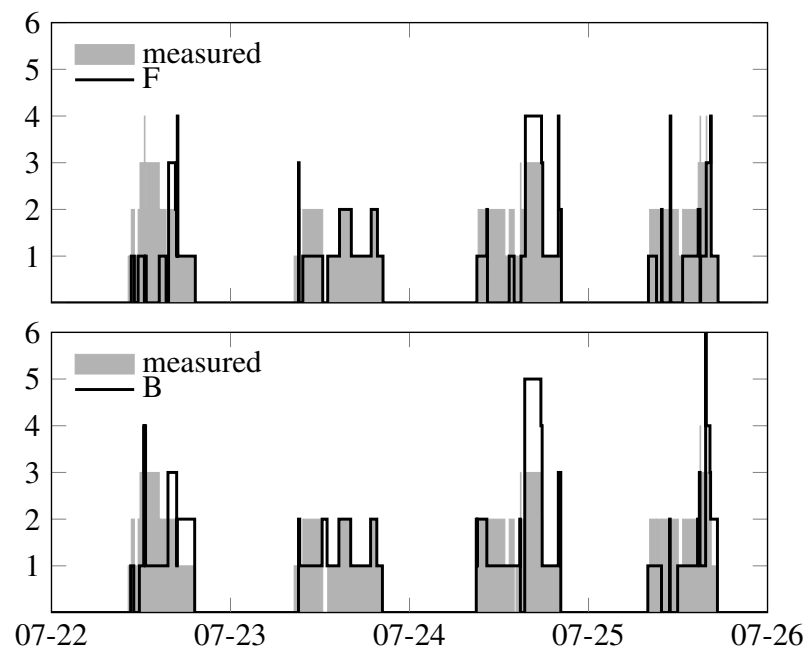

Fig. 2. Comparison of the estimates obtained combining the two possible solutions for the blind identification tier, i.e., estimators (11) and (16), with strategy (24) for solving the non-identifiability issue determined by our apriori belief $\gamma_{\max }=3$.

Table I then reports quantitatively the performance indexes achieved by the complete estimator, splitting the results for the two different strategies (11) and (16). As noticed before, the estimators tend to have on the specific experiment in consideration good detections of unoccupied rooms. 


\begin{tabular}{ccccc}
\hline Estimator & MSE & Accuracy & FP & FN \\
\hline F & 0.409 & 0.830 & 0.000 & 0.285 \\
B & 0.351 & 0.821 & 0.007 & 0.121 \\
\hline
\end{tabular}

TABLE I

SUMMARY OF THE PERFORMANCE INDEXES OF THE COMPLETE ESTIMATORS FOR THE TWO CASES OF SOLVING THE BLIND IDENTIFICATION PROBLEM WITH (11) OR (16).

\section{CONCLUSIONS}

We presented a strategy for the estimation of occupancy levels in the built environment that satisfies some practical needs: $i$ ) it is data-driven, so that it does not require apriori knowledges of the physics of the rooms under considerations; ii) it processes just $\mathrm{CO}_{2}$ concentration and $\mathrm{HVAC}$ actuation levels, information that is commonly available in modern buildings; iii) it does not require to perform training steps and collect pilot data with measurements of the actual occupancy.

The overall estimation strategy cascades a blind system identification step with an ad-hoc solution for removing ambiguities in the estimates. More specifically, we proposed two different ways to solve the blind identification step: i) a frequentist procedure assuming ARX-type dynamics with deterministic parameters, and estimating these parameters following ML concepts using non-linear optimization or alternated approach; ii) a Bayesian marginal likelihood scheme, where the impulse responses have a Gaussian prior, and their estimation obtained by implementing an EM algorithm.

The strategy was tested on a benchmark dataset, and was numerically shown to be effective. This suggests that, at least in environments similar to the one we used for our tests, there is a potential for blind identification strategies. This implicitly means that there are cases where it is possible to separate the dynamics of the environment from their inputs just by exploiting their fundamental structures and commonly available signals. We remark that the proposed strategy is still in its infancy, and it should be studied more. We devise future works specially in the analysis of its sensitivity to the various parameters and on the dataset on which it is applied.

\section{REFERENCES}

[1] F. Oldewurtel, D. Sturzenegger, and M. Morari, "Importance of occupancy information for building climate control," Applied Energy, vol. 101, pp. 521-532, Jan. 2013.

[2] D. Sturzenegger, F. Oldewurtel, and M. Morari, "Importance of LongTerm Occupancy Information - A Validation with Real Occupancy Data," in Clima - RHEVA World Congress, 2013.

[3] T. A. Nguyen and M. Aiello, "Energy intelligent buildings based on user activity: A survey," Energy \& Buildings, vol. 56, pp. 244-257, 2013.

[4] M. Gruber, A. Trüschel, and J.-O. Dalenbäck, "CO2 sensors for occupancy estimations: Potential in building automation applications," Energy and Buildings, vol. 84, pp. 548-556, Dec. 2014.

[5] N. Li, G. Calis, and B. Becerik-Gerber, "Measuring and monitoring occupancy with an RFID based system for demand-driven HVAC operations," Automation in Construction, vol. 24, pp. 89-99, July 2012.
[6] S. Meyn, A. Surana, Y. Lin, S. M. Oggianu, S. Narayanan, and T. A. Frewen, "A Sensor-Utility-Network Method for Estimation of Occupancy in Buildings," in IEEE Conference on Decision and Control, 2009, pp. 1494-1500.

[7] E. Hailemariam and R. Goldstein, "Real-time occupancy detection using decision trees with multiple sensor types," in Symposium on Simulation for Architecture and Urban Design, 2011.

[8] W. Kleiminger, C. Beckel, T. Staake, and S. Santini, "Occupancy Detection from Electricity Consumption Data," in Proceedings of the 5th ACM Workshop on Embedded Systems For Energy-Efficient Buildings - BuildSys'13. Rome, Italy: ACM Press, 2013, pp. 1-8.

[9] K. P. Lam, M. Höynck, B. Dong, B. Andrews, Y.-s. Chiou, D. Benitez, and J. Choi, "Occupancy detection through an extensive environmental sensor network in an open-plan office building," in IBPSA Conference, 2009, pp. 1452-1459.

[10] A. Ebadat, G. Bottegal, D. Varagnolo, B. Wahlberg, and K. H. Johansson, "Estimation of building occupancy levels through environmental signals deconvolution," in ACM Workshop On Embedded Systems For Energy-Efficient Buildings, 2013.

[11] B. Ai, Z. Fan, and R. X. Gao, "Occupancy Estimation for Smart Buildings by an Auto-Regressive Hidden Markov Model," in American Control Conference, 2014, pp. 2234-2239.

[12] R. H. Dodier, G. P. Henze, D. K. Tiller, and X. Guo, "Building occupancy detection through sensor belief networks," Energy and Buildings, vol. 38, no. 9, pp. 1033-1043, Sept. 2006.

[13] Z. Han, R. Gao, and Z. Fan, "Occupancy and indoor environment quality sensing for smart buildings," in Instrumentation and Measurement Technology Conference, May 2012, pp. 882-887.

[14] R. Melfi, B. Rosenblum, B. Nordman, and K. Christensen, "Measuring building occupancy using existing network infrastructure," in 2011 International Green Computing Conference and Workshops, July 2011, pp. 1-8.

[15] K. Abed-Meraim, W. Qiu, and Y. Hua, "Blind system identification," Proceedings of the IEEE, vol. 85, no. 8, 1997.

[16] G. Mustafaraj, J. Chen, and G. Lowry, "Development of room temperature and relative humidity linear parametric models for an open office using BMS data," Energy and Buildings, vol. 42, no. 3, pp. 348-356, 2010.

[17] D. Loveday and C. Craggs, "Stochastic modelling of temperatures for a full-scale occupied building zone subject to natural random influences," Applied Energy, vol. 45, no. 4, pp. 295-312, 1993.

[18] G. Lowry and M.-W. Lee, "Modelling the passive thermal response of a building using sparse BMS data," Applied Energy, vol. 78, no. 1, pp. 53-62, 2004.

[19] J. C.-M. Yiu and S. Wang, "Multiple ARMAX modeling scheme for forecasting air conditioning system performance," Energy Conversion and Management, vol. 48, pp. 2276-2285, 2007.

[20] S. Wu and J.-Q. Sun, "A physics-based linear parametric model of room temperature in office buildings," Building and Environment, vol. 50, pp. 1-9, Apr. 2012.

[21] F. Scotton, L. Huang, S. A. Ahmadi, and B. Wahlberg, "Physics-based Modeling and Identification for HVAC Systems," in European Control Conference, 2013.

[22] J. a. Tropp, I. S. Dhillon, R. W. Heath, and T. Strohmer, "Designing structured tight frames via an alternating projection method," IEEE Transactions on Information Theory, vol. 51, no. 1, pp. 188-209, 2005.

[23] A. Gunawardana and W. Byrne, "Convergence Theorems for Generalized Alternating Minimization Procedures," Journal of Machine Learning Research, vol. 6, pp. 2049-2073, 2005.

[24] G. Pillonetto, A. Chiuso, and G. De Nicolao, "Prediction error identification of linear systems: a nonparametric Gaussian regression approach," Automatica, vol. 47, no. 2, pp. 291-305, 2011.

[25] G. Pillonetto and G. De Nicolao, "A new kernel-based approach for linear system identification," Automatica, vol. 46, no. 1, pp. 81-93, 2010.

[26] T. Chen, H. Ohlsson, and L. Ljung, "On the estimation of transfer functions, regularizations and Gaussian processes - Revisited," Automatica, vol. 48, no. 8, pp. 1525-1535, Aug. 2012.

[27] G. Bottegal, R. S. Risuleo, and H. Hjalmarsson, "Blind system identification using kernel-based methods," arXiv preprint arXiv:1412.4056, 2014. 